\title{
Asymptotic Optimal Empirical Bayes Estimation of the Parameter of ЭРланга Distribution
}

\author{
Guobing Fan \\ Department of Basic Subjects, Hunan University of Finance and Economics, Changsha, China \\ Email address: \\ fanguobingmath@163.com \\ To cite this article: \\ Guobing Fan. Asymptotic Optimal Empirical Bayes Estimation of the Parameter of ЭРланга Distribution. American Journal of Applied \\ Mathematics. Vol. 4, No. 6, 2016, pp. 283-288. doi: 10.11648/j.ajam.20160406.14
}

Received: October 6, 2016; Accepted: October 14, 2016; Published: November 7, 2016

\begin{abstract}
This paper aims to study the empirical Bayes estimation of the parameter of ЭРланга distribution under a weighted squared error loss function. Bayes estimator is firstly to derive based on pivot method. Then empirical Bayes estimator of unknown parameter is constructed in a priori unknown circumstances. The asymptotically optimal property of this empirical Bayes estimator is also discussed. It is shown that the convergence rates of the proposed empirical Bayes estimator can arbitrarily close to $\left.\mathrm{O}(n)^{-1}\right)$ under suitable conditions.
\end{abstract}

Keywords: Empirical Bayes Estimator, Asymptotic Optimality, Weighted Squared Error Loss Function, ЭРланга Distribution

\section{Introduction}

Empirical Bayes method is firstly proposed by Robbins in 1956, and since then it becomes a very important statistical inference, which be applied to many fields, such as reliability, lifetime prediction, medical research, ect. [1-6]. Empirical Bayes estimations of various statistical models have received great attention. For example, Fan et al. [7] constructed a empirical Bayes estimator of the scale exponential family in the case of identically distributed and positively associated samples under weighted square loss function.

Rousseau and Szabo [8] considered the asymptotic behavior of the marginal maximum likelihood empirical Bayes posterior distribution in general setting. Wang and Wei [9] studied the empirical Bayes estimation problem for the scale-exponential family with errors in variables under the weighted square loss. Liu and Cao [10] discussed the empirical Bayes estimation about location parameter of twoexponential distribution under a LINEX loss function.in case of the negatively associated samples with kernel density method to estimate marginal probability density function. Zhang and Wei [11] first derived the Bayes estimators of variance components for one-way classification random effect model under the weighted square loss function, and then they constructed the empirical Bayes estimators by the kernel estimation method. Seal and Hossain [12] not only used EM algorithm to compute empirical Bayes estimates for different hyperparameters, but also investigated the robustness of empirical Bayes procedure. Steorts and Ghosh [13] considered benchmarked empirical Bayes estimators under the basic area-level model of Fay and Herriot while requiring the standard benchmarking constraint. Jiang [14] studied a monotone regularized kernel general empirical Bayes method for the estimation of a vector of normal means. Park [15] discussed the problem of simultaneous Poisson mean vector estimation and studied the performance of nonparametric empirical Bayes estimator from the view point of risk consistency.

ЭРланга distribution is an important distribution to model the repair time and guarantee the distribution delay time [16]. Pan et al. [17] studied the interval estimation and hypothesis test problem of ЭРланга distribution in case of small sample. Long [18] discussed the estimation of Зрланга distribution based on missing data.

Assume that random variable $X$ has the ЭРланга distribution with the following probability density function and cumulative distribution function, respectively:

$$
\begin{gathered}
f(x ; \delta)=4 x \delta^{2} e^{-2 \delta x}, \quad x \geq 0, \delta>0 \\
F(x ; \delta)=1-(1+2 \delta x) e^{-2 \delta x}, \quad x \geq 0, \delta>0
\end{gathered}
$$

Let $\theta=\delta / 2$, then the probability density function of 
ЭРланга distribution is

$$
f(x ; \theta)=x \theta^{2} e^{-\theta x}, \quad x \geq 0, \theta>0
$$

Here, $\theta$ is the unknown parameter, $\Omega=\{x \mid x>0\}$ is the sample space, and $\Theta=\{\theta \mid \theta>0\}$ is the parameter space.

This paper will study the empirical Bayes estimation of ЭРланга distribution in Eq. (3) under a weighted squared error loss function. The asymptotically optimal and its convergence rate of constructed empirical Bayes estimator will also be derived under certain suitable conditions. The remains of this paper are organized as follows: Section 2 will derive a Bayes estimator under weighted squared error loss function. Then Section 3 will put forward a new construction of empirical Bayes estimator using the pivot method. Section 4 studies the asymptotic optimality property of the proposed empirical Bayes estimator. Finally, Section 5 will give the conclusion of this paper.

\section{Bayes Estimation}

This section will study the Bayes estimation of ЭРланга distribution under the following weighted squared error loss function:

$$
L(\theta, \delta)=\frac{(\theta-\delta)^{2}}{\theta^{2}}
$$

The advantage of using the weighted square loss function is that it is invariant, and the Bayes expression is more concise, and the empirical Bayes estimation function is easy to construct. Under this loss function (4), there are also many authors discussed the Bayes estimation problem for different models[19-24].

In the following discussion, we always assume:

The parameter $\theta$ has the prior distribution function $G(\theta)$, which is unknown and belongs to the below set:

$$
F=\left\{G(\theta): E\left(\theta^{2}\right)<\infty, E(\theta)<\infty, E\left(\theta^{-1}\right)<\infty\right\}
$$

Under the weighted squared error loss function (3), the Bayes estimator $\delta_{G}(x)$ of the parameter $\theta$ relative to the prior $G(\theta)$ is given by

$$
\delta_{G}(x)=\frac{E\left(\theta^{-1} \mid x\right)}{E\left(\theta^{-2} \mid x\right)}=\frac{p_{1}(x)}{p_{2}(x)}
$$

Where

$$
\begin{aligned}
& p_{1}(x)=\int_{\Theta} x \theta e^{-\theta x} d G(\theta) \\
& p_{2}(x)=\int_{\Theta} x e^{-\theta x} d G(\theta)
\end{aligned}
$$

The Bayes risk associated with $\delta_{G}(x)$ is

$$
R_{G}=R\left(\delta_{G}, G\right)=\inf _{\delta} R(\delta, G)=E_{(X, \theta)}\left[\frac{(\theta-\delta)^{2}}{\theta^{2}}\right]
$$

Here $E_{(X, \theta)}[\cdot]$ denotes the expectation with respect to the joint distribution of $(X, \theta)$.

Remark 1. Note that, if the prior distribution $G(\theta)$ is known, $\delta(x)$ equals to $\delta_{G}(x)$, we can get $R_{G}$. Unfortunately here we assume the prior distribution $G(\theta)$ is unknown, then $\delta_{G}(x)$ is unknown too. Then the Bayes estimator $\delta_{G}(x)$ is no practical value, therefore, we need to introduce the empirical Bayes method, which requires construct the risk function can be arbitrary close to $R_{G}$.

\section{The Construction of Empirical Bayes Estimation}

Suppose that $\left(X_{1}, \theta_{1}\right),\left(X_{2}, \theta_{2}\right), \ldots, \quad\left(X_{n}, \theta_{n}\right) \quad$ and $\left(X_{n+1}, \theta_{n+1}\right) \triangleq(X, \theta)$ are independent identical distribution random samples, where $X_{1}, X_{2}, \ldots, X_{n}$ are the historical samples, and $X$ is the present sample. Here $\theta_{i}, i=1, \cdots, n$ and $\theta$ have the same prior distribution. $X_{i}, i=1, \cdots, n$ and $X$ have the same marginal probability density function $f_{G}(x)$.

Lemma 1 let $X_{1}, \cdots, X_{n}$ be the independent identical distribution random samples distributed with ЭРланга distribution (1). Define

$$
\begin{gathered}
V_{j}(x)=\frac{x}{X_{j}} I\left(X_{j}-x\right), \\
p_{3}(x)=\int_{\Theta} \theta^{-3} x \theta^{2} e^{-\theta x} d G(\theta)=\int_{\Theta} x \theta^{-1} e^{-\theta x} d G(\theta), \\
p_{1 n}(x)=\frac{1}{n} \sum_{j=1}^{n} V_{j}(x) \\
p_{2 n}(x)=\frac{1}{n} \sum_{j=1}^{n}\left[\left(X_{j}-x\right) V_{j}(x)\right]
\end{gathered}
$$

Then

(i) $p_{1 n}(x)$ and $p_{2 n}(x)$ are the unbiased estimator of $p_{1}(x)$ and $p_{2}(x)$,respectively. That is

$$
E\left(p_{1 n}(x)\right)=p_{1}(x), E\left(p_{2 n}(x)\right)=p_{2}(x)
$$

(ii) $\operatorname{Var}\left(p_{1 n}(x)\right) \leq \frac{1}{n} p_{1}(x), \operatorname{Var}\left(p_{2 n}(x)\right) \leq \frac{2}{n} p_{3}(x)$

Proof. (i) By Eq. (3), the marginal probability density function $f_{G}(x)$ is

$$
f_{G}(x)=\int_{\Theta} f(x \mid \theta) d G(\theta)=\int_{\Theta} x \theta^{2} e^{-\theta x} d G(\theta)
$$

By Eq.(10) and Eq.(14), 


$$
\begin{aligned}
& E V_{j}(x)=\int_{x}^{\infty} \frac{x}{t} f_{G}(t) d t \\
&=\int_{x}^{\infty} \frac{x}{t}\left[\int_{\Theta} t \theta^{2} e^{-\theta t} d G(\theta)\right] d t \\
&=\int_{x}^{\infty}\left[\int_{\Theta} x \theta^{2} e^{-\theta t} d G(\theta)\right] d t \\
&=\int_{\Theta}\left[\int_{x}^{\infty} e^{-\theta t} d t\right] x \theta^{2} d G(\theta) \\
&=\int_{\Theta} \frac{1}{\theta} x \theta^{2} e^{-\theta x} d G(\theta) \\
&=E\left(\frac{1}{\theta} \mid x\right)=p_{1}(x)
\end{aligned}
$$

Note that $V_{j}(x), j=1, \cdots, n$ is an independent identical distribution random sequence. Then

$$
\begin{aligned}
& E\left(p_{1 n}(x)\right)=E\left[\frac{1}{n} \sum_{j=1}^{n} V_{j}(x)\right]=E V_{j}(x)=p_{1}(x) \\
& E\left(X_{j}-x\right) V_{j}(x) \\
& =\int_{x}^{\infty}(t-x) \frac{x}{t}\left[\int_{\Theta} \theta^{2} t e^{-\theta t} d G(\theta)\right] d t \\
& =\int_{x}^{\infty}\left[(t-x) x \int_{\Theta} \theta^{2} e^{-\theta t} d G(\theta)\right] d t \\
& =\int_{\Theta}\left[\int_{x}^{\infty} t e^{-\theta t} d t\right] x \theta^{2} d G(\theta) \\
& \quad-\int_{\Theta}\left[\int_{x}^{\infty} e^{-\theta t} d t\right] x^{2} \theta^{2} d G(\theta) \\
& =\int_{\Theta}\left(\frac{x}{\theta}+\frac{1}{\theta^{2}}\right) e^{-\theta x} x \theta^{2} d G(\theta) \\
& \quad-\int_{\Theta} \frac{1}{\theta} e^{-\theta x} x^{2} \theta^{2} d G(\theta) \\
& =E\left(\frac{1}{\theta^{2}} \mid x\right)=p_{2}(x)
\end{aligned}
$$

The random sequence $\left(X_{j}-x\right) V_{j}(x), j=1, \cdots, n$ is also an independent identical distribution random sequence. Then

$$
E\left(p_{2 n}(x)\right)=E\left[\frac{1}{n} \sum_{j=1}^{n}\left(X_{j}-x\right) V_{j}(x)\right]=p_{2}(x)
$$

For the case (ii).

$$
\begin{aligned}
& \operatorname{Var}\left(V_{j}(x)\right)=E V_{j}^{2}(x)-\left[E V_{j}(x)\right]^{2} \\
& =\int_{x}^{\infty} \frac{u^{2}(x)}{u^{2}(t)}\left[\int_{\Theta} t \theta^{2} e^{-\theta t} d G(\theta)\right] d t-P_{1}^{2}(x) \\
& =\int_{\Theta}\left[\int_{x}^{\infty} \frac{1}{t} e^{-\theta t} d t\right] x^{2} \theta^{2} d G(\theta)-P_{1}^{2}(x) \\
& \leq \int_{\Theta}\left[\int_{x}^{\infty} e^{-\theta t} d t\right] x \theta^{2} d G(\theta)-P_{1}^{2}(x)
\end{aligned}
$$

$$
\begin{aligned}
& =\int_{\Theta} \frac{1}{\theta} e^{-\theta x} x \theta^{2} d G(\theta)-p_{1}^{2}(x) \\
& =p_{1}(x)-p_{1}^{2}(x) \leq p_{1}(x)
\end{aligned}
$$

Because $V_{j}(x), j=1, \cdots, n$ is an independent identical distribution random sequence. Then

$$
\begin{gathered}
\operatorname{Var}\left(p_{1 n}(x)\right) \leq \frac{1}{n} p_{1}(x) \\
\operatorname{Var}\left(\left(X_{j}-x\right) V_{j}(x)\right) \leq E\left(\left(X_{j}-x\right) V_{j}(x)\right)^{2} \\
=\int_{x}^{\infty}(t-x)^{2} \frac{x^{2}}{t^{2}}\left[\int_{\Theta} t \theta^{2} e^{-\theta t} d G(\theta)\right] d t \\
\int_{\Theta}\left[\int_{x}^{\infty} \frac{(t-x)^{2}}{t} e^{-\theta t} d t\right] x^{2} \theta^{2} d G(\theta) \\
\leq \int_{\Theta}\left[\int_{x}^{\infty}(t-x)^{2} e^{-\theta t} d t\right] x \theta^{2} d G(\theta) \\
=\int_{\Theta} \frac{2}{\theta^{3}} e^{-\theta x} x \theta^{2} d G(\theta)=2 p_{3}(x)
\end{gathered}
$$

Similarly, it is easy to verify that

$$
\operatorname{Var}\left(p_{2 n}(x)\right) \leq \frac{2}{n} p_{3}(x) .
$$

Then the Lemma 1 is proved.

Definition 1. According to Eq. (6) and utilizing $p_{1 n}(x)$ and $p_{2 n}(x)$, this paper constructs an empirical Bayes estimator of the parameter $\theta$ of ЭРланга distribution as follows.

$$
\delta_{n}(x)=\frac{p_{1 n}(x)}{\hat{p}_{2 n}(x)}
$$

Where

$$
\hat{p}_{2 n}(x)=\left[p_{2 n}(x)\right]_{n^{v}}
$$

and $[u]_{c}=\left\{\begin{array}{cl}-c & , u<-c \\ u & ,|u| \leq c \\ c & , u>c\end{array}\right.$

\section{Asymptotic Optimal Properties of the Emprical Bayes Estimator}

This section will study the asymptotic optimality property of the proposed empirical Bayes estimator. Under the weighted squared error loss, the Bayes risks of the proposed empirical Bayes estimator $\delta_{n}(x)$ is

$$
R_{n}=R_{n}\left(\delta_{n}, G\right)=E_{*}\left[\frac{\left(\theta-\delta_{n}\right)^{2}}{\theta^{2}}\right]
$$

Here $E_{*}[\cdot]$ denotes the expectation with respect to the joint 
distribution of $\left(X_{1}, \ldots, X_{n},(X, \theta)\right)$. In the following discussion, we suppose that $E[\cdot]$ denotes the expectation with respect to the joint distribution of $\left(X_{1}, \ldots, X_{n}\right)$.

Definition 2. If $\lim _{n \rightarrow \infty} R\left(\delta_{n}(x), G(\theta)\right)=R_{G}$, then it means that the random variable sequence $\left\{\delta_{n}(x)\right\}$ is a asymptotic optimality empirical Bayes estimators sequence.

Further, if $R\left(\delta_{n}(x), G(\theta)\right)-R_{G}=\mathrm{O}\left(n^{-q}\right), q>0$, then it is to say the convergence rate of the estimators sequence $\left\{\delta_{n}(x)\right\}$ is $\mathrm{O}\left(n^{-q}\right)$.

According to Definition 2, we can use the nonnegative difference between $R\left(\delta_{n}(x), G(\theta)\right)$ with $R_{G}$ as the measure to evaluate the performance of the proposed empirical Bayes estimator $\delta_{n}(x)$

In the following discussion, we always assume $c, c_{1}, c_{2}, \ldots$ represent different constants, even they occur in the same expression they also may take different values.

Lemma 2 [25] Assume that $\delta$ is an arbitrary estimator of parameter $\theta$, then

$$
R(\delta, G)-R_{G}=E_{*}\left[\frac{\left(\delta-\delta_{n}\right)^{2}}{\theta^{2}}\right],
$$

Where $R(\delta, G)$ represents the Bayesian risk of estimator $\delta$

Theorem 1 Suppose $R_{n}$ and $R_{G}$ definite in Eq. (17) and Eq. (9) separately, then for ЭРланга distribution, if prior distribution $G(\theta)$ belongs to $F$ defined in Eq. (5), then

$$
\lim _{n \rightarrow \infty} R\left(\delta_{n}(x), G(\theta)\right)=R_{G}
$$

Proof. According to Lemma2,

$$
\begin{aligned}
& R\left(\delta_{n}(x), G(\theta)\right)-R_{G}=E_{*}\left[\frac{\left(\delta_{B}-\delta_{n}\right)^{2}}{\theta^{2}}\right] \\
& =E_{(X, \theta)}\left[\theta^{-2} E\left(\delta_{n}-\delta_{B}\right)^{2}\right]
\end{aligned}
$$

By the control convergence theorem, to prove the theorem we need only prove the following conclusions.

(i) For any given $x$ and $\theta, \lim _{n \rightarrow \infty} \theta^{-2} E\left(\delta_{n}-\delta_{B}\right)^{2}=0$.

(ii) $\theta^{-2} E\left(\delta_{n}-\delta_{B}\right)^{2} \leq M(x, \theta)$, for a sufficiently large $n$ and $E_{(X, \theta)} M(x, \theta)<\infty$

Now we prove these two cases.

$$
\begin{aligned}
& E\left(\delta_{n}-\delta_{B}\right)^{2} \\
& =E\left(\frac{p_{1 n}(x)}{\hat{p}_{2 n}(x)}-\frac{p_{1}(x)}{p_{2}(x)}\right)^{2} \\
& =E\left[\frac{p_{1 n}(x)-p_{1}(x)}{\hat{p}_{2 n}(x)}-\left(\frac{p_{1}(x)}{p_{2}(x)}-\frac{p_{1}(x)}{\hat{p}_{2 n}(x)}\right)\right]^{2}
\end{aligned}
$$

$$
\begin{aligned}
& =E\left[\frac{p_{1 n}(x)-p_{1}(x)}{\hat{p}_{2 n}(x)}-\delta_{B}\left(1-\frac{p_{2}(x)}{\hat{p}_{2 n}(x)}\right]^{2}\right. \\
& \leq 2 E\left[\frac{p_{1 n}(x)-p_{1}(x)}{\hat{p}_{2 n}(x)}\right]^{2}+2 \delta_{B}^{2} E\left[1-\frac{p_{2}(x)}{\hat{p}_{2 n}(x)}\right]^{2}
\end{aligned}
$$

That is

$$
E\left(\delta_{n}-\delta_{B}\right)^{2} \leq 2\left(I_{1}+\delta_{B}^{2} I_{2}\right)
$$

Where

$$
\begin{gathered}
I_{1}=E\left[\frac{p_{1 n}(x)-p_{1}(x)}{\hat{p}_{2 n}(x)}\right]^{2}, \\
I_{2}=E\left[1-\frac{p_{2}(x)}{\hat{p}_{2 n}(x)}\right]^{2} .
\end{gathered}
$$

Then by Lemma 1, we have

$$
\begin{aligned}
I_{1} & \leq \delta_{n}^{-2} E\left[p_{1 n}(x)-p_{1}(x)\right]^{2} \\
& =\delta_{n}^{-2} \operatorname{Var}\left(p_{1 n}(x)\right) \\
& \leq \frac{1}{n} \delta_{n}^{-2} p_{1}(x)
\end{aligned}
$$

$$
\begin{aligned}
I_{2} \leq & E\left[\frac{\hat{p}_{2 n}(x)-p_{2}(x)}{\hat{p}_{2 n}(x)}\right]^{2} I\left(p_{2}(x) \geq \delta_{n}\right) \\
& +E\left[\frac{\hat{p}_{2 n}(x)-p_{2}(x)}{\hat{p}_{2 n}(x)}\right]^{2} I\left(p_{2}(x)<\delta_{n}\right) \\
= & J_{1}+J_{2}
\end{aligned}
$$

Here

$$
\begin{aligned}
& J_{1}=E\left[\frac{\hat{p}_{2 n}(x)-p_{2}(x)}{\hat{p}_{2 n}(x)}\right]^{2} I\left(p_{2}(x) \geq \delta_{n}\right), \\
& J_{2}=E\left[\frac{\hat{p}_{2 n}(x)-p_{2}(x)}{\hat{p}_{2 n}(x)}\right]^{2} I\left(p_{2}(x)<\delta_{n}\right) .
\end{aligned}
$$

Obviously that

$p_{2}(x)-\hat{p}_{2 n}(x)= \begin{cases}p_{2}(x)-\hat{p}_{2 n}(x), & ,\left|p_{2 n}(x)\right| \geq \delta_{n} \\ p_{2}(x)-\delta_{n}<p_{2}(x)-\hat{p}_{2 n}(x), & \left|p_{2 n}(x)\right|<\delta_{n}\end{cases}$

Then

$$
\begin{gathered}
J_{1} \leq \delta_{n}^{-2} E\left[\hat{p}_{2 n}(x)-p_{2}(x)\right]^{2} I\left(p_{2}(x) \geq \delta_{n}\right) \\
\leq \delta_{n}^{-2} E\left[p_{2 n}(x)-p_{2}(x)\right]^{2} \\
=\delta_{n}^{-2} \operatorname{Var}\left(p_{2 n}(x)\right) \\
\leq \frac{1}{n} \delta_{n}^{-2} p_{3}(x)
\end{gathered}
$$

If $p_{2}(x)<\delta_{n}$, then $\left|\frac{p_{2}(x)}{\hat{p}_{2 n}(x)}\right| \leq 1$, further 


$$
J_{2} \leq 4 \delta_{n} I\left(p_{2}(x)<\delta_{n}\right)
$$

By the inequalities (19)-(21), we have

$$
\begin{aligned}
\theta^{-2} E\left(\delta_{n}-\delta_{B}\right)^{2} \leq & \frac{2}{n} \delta_{n}^{-2} p_{1}(x) \theta^{-2}+\frac{2}{n} \delta_{n}^{-2} p_{3}(x) \\
& +8 \delta_{n} I\left(p_{2}(x)<\delta_{n}\right)
\end{aligned}
$$

Then for any given $x$ and $\theta$,

$$
\lim _{n \rightarrow \infty} \theta^{-2} E\left(\delta_{n}-\delta_{B}\right)^{2}=0
$$

Thus the case (i) is true.

For the case (ii), by inequality (22),

$$
\theta^{-2} E\left(\delta_{n}-\delta_{B}\right)^{2} \leq p_{1}(x) \theta^{-2}+p_{3}(x) \theta^{-2} \triangleq M(x, \theta)
$$

Further

$$
\begin{aligned}
p_{1}(x) & =\int_{\Theta} x \theta e^{-\theta x} d G(\theta) & p_{3}(x) & =\int_{\Theta} \theta^{-1} x e^{-\theta x} d G(\theta) \\
& \leq x \int_{\Theta} \theta d G(\theta) & & \leq x \int_{\Theta} \theta^{-1} d G(\theta) \\
& =u(x) E\left(\theta^{-1} c(\theta)\right)<\infty & & =x E\left(\theta^{-1}\right)<\infty
\end{aligned}
$$

Then the case (ii) is true.

Thus we finish the proof of the theorem.

\section{Conclusions}

For the estimation of the parameter of ЭРланга distribution, this paper puts forward an empirical Bayes approach to estimate the parameter under a weighted squared error loss function when the prior distribution of the parameter is unknown.. The asymptotically optimal property of the proposed empirical Bayes estimator is also discussed. The convergence rates of the proposed empirical Bayes estimator can arbitrarily close to $\mathrm{O}\left(n^{-1}\right)$ under suitable conditions. The proposed method can be similarly extended to the construction of empirical Bayesian estimator of other distributions, such as Rayleigh distribution, Lomax distribution, etc.

\section{Acknowledgement}

This study is partially supported by Natural Science Foundation of Hunan Province (No. 2016JJ4012 and No. 2015JJ3030) and Foundation of Hunan Educational Committee (No.15C0228). The author also gratefully acknowledges the helpful comments and suggestions of the reviewers, which have improved the presentation.

\section{References}

[1] Clayton D., Kaldor J., 1987. Empirical Bayes estimates of age-standardized relative risks for use in disease mapping. Biometrics, 43(3):671-81.

[2] Karunamuni R. J., 1996. Empirical Bayes sequential estimation for exponential families: the untruncated component. Annals of the Institute of Statistical Mathematics, 48(4): 711-730.

[3] Guarino C. M., Maxfield M., Reckase M. D., et al., 2015. An evaluation of empirical Bayes's estimation of value-added teacher performance measures. Journal of Educational \& Behavioral Statistics, 40(2):190-222.

[4] Prakash G., 2015. Reliability performances based on empirical Bayes censored Gompertz data. International Journal of Advanced Research, 3(11):1297-1307.

[5] Coram M., Candille S., Duan, Q., Chan K. H., Li Y., Kooperberg C., et al., 2015. Leveraging multi-ethnic evidence for mapping complex traits in minority populations: an empirical Bayes approach. American Journal of Human Genetics, 96(5): 740-52.

[6] Naznin F., Currie G., Sarvi M., Logan D., 2015. An empirical Bayes safety evaluation of tram/streetcar signal and lane priority measures in melbourne. Traffic Injury Prevention, 17(1): 91-97.

[7] Fan G. L., Ling N. X., Xu H. X., 2010. Asymptotically optimal empirical Bayes estimation of parameter for scaleexponential family under PA Samples. Chinese Quarterly Journal of Mathematics, 25(3):372-378.

[8] Rousseau J., Szabo B., 2015. Asymptotic behaviour of the empirical Bayes posteriors associated to maximum marginal likelihood estimator. Statistics, 40(10):885-9.

[9] Wang L., Wei L., 2010. Empirical Bayes estimation for the scale parameter with errors in variables. Southeast Asian Bulletin of Mathematics, 34(6):1119-1131.

[10] Liu R., Cao Y., 2011. Optimal behavior of EB estimator for two-exponential distribution family. Journal of Huazhong Normal University, 45(4):542-546.

[11] Zhang W. P., Wei L. S., 2005. The asymptotically optimal empirical Bayes estimators of variance components in oneway classification random effect model. Journal of Systems Science and Mathematical Sciences, 25(1):106-117.

[12] Seal B., Hossain S. J., 2015. Empirical Bayes estimation of parameters in Markov transition probability matrix with computational methods. Journal of Applied Statistics, 42(42):508-519.

[13] Steorts R. C., Ghosh, M., 2013. On estimation of mean squared errors of benchmarked empirical Bayes estimators. Statistica Sinica, 23(2): 749-767.

[14] Jiang W., 2013. On regularized general empirical Bayes estimation of normal means. Journal of Multivariate Analysis, 114(2):54-62.

[15] Park J. Y., 2012. Nonparametric empirical Bayes estimator in simultaneous estimation of Poisson means with application to mass spectrometry data. Journal of Nonparametric Statistics, 24(1):245-265.

[16] Lv H. Q., Gao L. H. and Chen C. L., 2002. Эрланга distribution and its application in supportability data analysis. Journal of Academy of Armored Force Engineering, 16(3): 48-52.

[17] Pan G. T., Wang B. H., Chen C. L., Huang Y. B.and Dang M. T., 2009. The research of interval estimation and hypothetical test of small sample of Зрланга distribution. Application of Statistics and Management, 28(3): 468-472. 
[18] Long В., 2013. The estimations of parameter from Зрланга distribution under missing data samples. Journal of Jiangxi Normal University (Natural Science), 37(1): 16-19.

[19] Podder C. K., Roy M. K., Bhuiyan K. J., et al., 2004. Minimax estimation of the parameter of the Pareto distribution under quadratic and MLINEX loss functions. Pakistan Journal of Statistics, 20(1):137-149.

[20] Yang L. W., 2013. Minimax estimation of parameter for a class of scale distributions under different loss functions. Journal of Anhui University, 37(5):6-11.

[21] Ren H. P., Li J. P., 2012. Bayes estimation of traffic intensity in $\mathrm{M} / \mathrm{M} / 1$ queue under a new weighted square error loss function. Advanced Materials Research, 2012, 485:490-493.

[22] Bao X. U., Song L. X., 2009. Estimator of the scale-parameter in a class of the exponential family under weighted quadratic loss function. Journal of Natural Science of Heilongiiang University, 26(6):766-769.
[23] Li L. P., 2016. Minimax estimation of the parameter of ЭРланга distribution under different loss functions, Science Journal of Applied Mathematics and Statistics. 4(5): 229-235.

[24] Yi W., Yan B., Miu B. Q. Y., 2012. Foreign exchange rates prediction based on generalized exponential predictor models with weighted loss function. Journal of Applied Statistics \& Management, 31(5):799-804

[25] Wang L. C., Wei L. S., 2002. The convergence rates of empirical Bayes estimation of the parameter for scaleexponential family. Chinese Annals of Mathematics Series A, 23(5): 555-564.

[26] Wang L. C., Wei L. S., 2002. Asymptotically optimal empirical Bayes estimation for parameters of scaleexponential families. Journal of University of Science and Technology of China, 32(1):62-69. 